\title{
Decoding Laboratory Diagnosis of Mycoplasma pneumoniae
}

\author{
Aruna Poojary
}

\begin{abstract}
Mycoplasma pneumoniae contributes to $30-40 \%$ of community-acquired pneumonia in children and has the potential to cause clusters and outbreaks in closed settings. Early diagnosis helps in appropriate management of the case and also reduces long-term complications associated with extrapulmonary disease. Molecular testing and specific serology remain the backbone of laboratory diagnosis for Mycoplasma pneumoniae. Pediatric Infectious Disease (2020): 10.5005/jp-journals-10081-1279
\end{abstract}

\section{INTRODUCTION}

Mycoplasmas are the smallest free living prokaryotic organisms. They are ubiquitous in nature and cause infections in humans, animals, insects, and plants. 'The genus Mycoplasma belongs to the class Mollicutes, family Mycoplasmataceae. ${ }^{2}$ Mycoplasmas lack a rigid cell wall; as a result, they are resistant to beta-lactam antimicrobial agents that act on the cell wall. While over 200 species of mycoplasmas have been described, only 17 cause human infections. ${ }^{1,3}$ Mycoplasmas are known to cause respiratory and genital infections. The most common and well-studied Mycoplasma causing human infections is Mycoplasma pneumoniae (MP) that causes both upper and lower respiratory disease in both children and adults.

\section{Need for Laboratory Confirmation of $\boldsymbol{M}$. PNEUMONIAE (MP)}

- Approximately $30-40 \%$ of community-acquired pneumonia in children and young adults is due to MP and it can be treated with antimicrobial agents. ${ }^{4}$

- It may also cause asthma-like exacerbations in young children and infants.

- These infections have an epidemic potential especially in households and schools.

- Mycoplasma pneumoniae causes disease similar to many respiratory viruses and therefore should be considered in the differential diagnosis and must be worked up with the help of lab tests. ${ }^{4}$

- The spectrum of infections caused by MP ranges from mild infections called "walking pneumonia" to severe pneumonia.

- Extrapulmonary manifestations of MP infection have been described in almost every organ system. ${ }^{5}$ These occur either due to the direct or indirect, i.e., antibody- or autoimmune-mediated mechansims, and are responsible for increased morbidity and mortality. ${ }^{6}$ Extrapulmonary manifestations often occur in the absence of obvious pneumonia or respiratory symptoms thus making the diagnosis further challenging.

- Pathogen-specific testing plays an important role in targeted therapy.

\section{Laboratory Diagnosis of M. pneumoniae}

Laboratory diagnosis of MP begins with keeping a high degree of suspicion as the organisms are fastidious and are likely to be missed
Department of Pathology and Microbiology, Breach Candy Hospital Trust, Mumbai, Maharashtra, India

Corresponding Author: Aruna Poojary, Department of Pathology and Microbiology, Breach Candy Hospital Trust, Mumbai, Maharashtra, India, Phone: +91 9820109602, e-mail: arunapoojary@gmail.com

How to cite this article: Poojary A. Decoding Laboratory Diagnosis of Mycoplasma pneumoniae. Pediatr Inf Dis 2020;2(4):158-160.

Source of support: Nil

Conflict of interest: None

otherwise. The investigations can be classified as those specific for MP (culture, serology, and PCR) and the nonspecific tests (cold agglutinin test, WBC counts, ESR).

\section{Type of Specimens Acceptable for Testing ${ }^{5}$}

- Respiratory specimens like throat swabs, nasopharyngeal swabs, throat washings, sputum, tracheal and transtracheal aspirates, bronchoalveolar lavage, pleural fluid, and lung biopsies are acceptable.

- Extrapulmonary specimens like CSF, pericardial fluid, tissue or skin lesions.

- Serum may be used for detection of specific antibodies against MP.

- EDTA blood may be used to perform cold agglutinin tests.

\section{Measures to Improve Recovery of MP from Various Specimens $^{5}$}

- Desiccation leads to loss of viability, hence use transport media especially for tissues to keep them moist.

- If the storage time of the specimen is more than 24 hours, use transport medium and freeze at $-80^{\circ} \mathrm{C}$.

- Frozen samples should be thawed in a water bath at $37^{\circ} \mathrm{C}$.

- Avoid cotton or calcium alginate swabs as they can be inhibitory to MP recovery, both in culture and PCR. Use of dacron or polyester swabs is recommended.

- Blood for PCR testing is collected in a vacutainer containing acid citrate dextrose

\section{Culture}

Mycoplasma pneumoniae have fastidious growth requirements and grow very slowly requiring up to 6 weeks of incubation

(C) The Author(s). 2020 Open Access This article is distributed under the terms of the Creative Commons Attribution 4.0 International License (https:// creativecommons.org/licenses/by-nc/4.0/), which permits unrestricted use, distribution, and non-commercial reproduction in any medium, provided you give appropriate credit to the original author(s) and the source, provide a link to the Creative Commons license, and indicate if changes were made. The Creative Commons Public Domain Dedication waiver (http://creativecommons.org/publicdomain/zero/1.0/) applies to the data made available in this article, unless otherwise stated. 
for growth. Cell-free formulations like Modified New York City medium, PPLO broth or agar with yeast extract and horse serum, soy peptone, or biphasic SP-4 medium have been used for culture. ${ }^{7}$ Colonies that grow on the agar surface need to undergo further testing preferably PCR-based tests to differentiate MP from other commensal Mycoplasmas. ${ }^{2}$ Collection of appropriate specimens and transportation to the lab is of utmost importance for isolation. Culture methods are insensitive and therefore not useful in routine clinical microbiology settings. ${ }^{8}$ However, culture remains an important tool for biological and molecular characterization of the isolates, which is required for research or epidemiological purposes. $^{8}$

\section{Advantages}

- Reference standard method for diagnosis of infection

- Helps in detection of antimicrobial resistance and further molecular research

\section{Disadvantages}

- Laborious and expensive ${ }^{7}$

- Not therapeutically relevant with respect to turnaround time

- Needs additional confirmation tests

\section{Molecular Testing}

Molecular testing can be of two types, i.e., hybridization assays or amplification (nucleic acid amplification, NAAT)-based techniques. It basically involves detection of MP nucleic acids (DNA or RNA) from respiratory specimens like nasopharyngeal swab/wash, oropharyngeal swab/wash, sputum, bronchoalveolar lavage, pleural fluid, lung biopsy, etc. ${ }^{7,8}$ In the past decade, the interest in nonculture-based methods of diagnosis has increased significantly.

- Hybridization assays: Hybridization with DNA probes was developed as early as 1980s and was the first molecular test for MP diagnosis. ${ }^{7}$ Hybridization can be performed on various respiratory specimens. The sensitivity and specificity of results in sputum was found to be $95 \%$ and $85 \%$, respectively. ${ }^{9}$ These assays have been replaced by amplification assays because of their increased sensitivity and also to avoid the handling of radiolabeled probes. ${ }^{1}$

- Nucleic acid amplification tests (NAATs): These are also called as polymerase chain reaction (PCR)-based tests or nucleic acid amplification technology (NAAT). The PCR is almost always more sensitive that the traditional serology-based tests and hence considered as the "new gold standard" in MP diagnosis. ${ }^{8}$ They are considered as a rapid diagnostic tool for MP infections. PCR is available as monoplex and multiplex PCRs. ${ }^{8}$ In monoplex PCRs, only a single target organism is detected in this case it is MP. Multiplex PCRs are often syndromic approach based, where multiple target organisms (bacteria, atypical bacteria, or viruses) are identified using a single test and a single specimen in a patient presenting with similar clinical manifestations, in this case either as upper or lower respiratory symptoms and signs. Multiplex PCRs are available in India, which offer as many as 22 targets along with Mycoplasma (Seegene, Fast Track diagnostics, Pathofinder, Biofire, and BD Max). Polymerase chain reactions have higher sensitivity as well as positive predictive value as compared to serological diagnosis. ${ }^{9}$
PCR diagnosis is not always sufficient for detecting Mycoplasma infections causing disease. Several studies have shown poor correlation between serology, culture, and PCR-based methods., This is because the presence of the pathogen does not always indicate disease as asymptomatic carriage is common in children. ${ }^{5}$ It is therefore important that clinical findings along with laboratory findings be considered in the diagnosis of MP.

\section{Advantages of Molecular Testing}

- Multiplex PCRs can identify one pathogen from a single specimen.

- Does not require viable organisms for detection.

- Availability of "Sample to answer" type of platform, which can give results within 1 hour.

- Analytical sensitivity of most assays is $<100$ genome copies. ${ }^{1}$

- These tests can be performed within the 1st week of illness when antibody response has not yet developed.

- They can also be performed on formalin fixed tissues and extrapulmonary samples like pericardial fluid, blood, cerebrospinal fluid, etc. ${ }^{6}$

\section{Disadvantages of Molecular Testing}

- Are not widely available in India

- Expensive especially multiplex PCRs

- May pick up nonviable organisms giving false-positive results.

\section{Antigen Detection}

Tests are available to detect the various antigens (e.g., ribosomal protein L7/L12) of MP based on immunological methods. Antigenbased tests are not widely available in India and have limited sensitivity and specificity. ${ }^{10,11}$

\section{Serology}

Antibodies usually start appearing soon after the 1st week of clinical illness, peak around 3-6 weeks, and then gradually decline over months to years. ${ }^{5}$ While both $\lg M$ and $\lg G$ antibodies can be detected, the presence of specific IgM antibodies against MP is considered diagnostic of recent infection. This has also been the most common diagnostic test used for many years. ${ }^{3} \mathrm{~A}$ fourfold rise in antibody titer when paired sera is collected at least 2 weeks apart is considered as a "gold standard" for diagnosis. Studies have shown that the likelihood of diagnosis improved significantly when instead of one positive antibody result (30\%), paired sera was used $(88.6 \%)$ for diagnosis. Unfortunately, in practice, we rarely receive paired sera and therefore one cannot rely upon single IgM results for diagnosis of MP infections. Serology plays a very important role in diagnosis especially if extrapulmonary manifestations are suspected in the absence of respiratory symptoms. ${ }^{6}$ In such cases, paired acute and convalescent sera should be tested using quantitative methods like particle agglutination or ELISA. ${ }^{6}$

\section{Advantages}

- Easily and widely available

- Relatively easy to perform or incorporate in the lab workflow as ELISA is a common procedure in in most labs

- Important role in extrapulmonary manifestations, which many a times occur without obvious pulmonary disease. 


\section{Disadvantages}

- Not detected within the 1st week of infection

- Antibody production is an indirect host response and many patients may not raise antibodies especially those with immunodeficiencies or immunosuppression. For those with high background IgG levels, estimating antibodies can lead to nonspecific results. ${ }^{5}$

- Serology tests are that they can cross-react with other species of Mycoplasma, e.g., M. genitalium leading to reduced specificity. ${ }^{4}$

- Specificity of different commercially available assays varies widely between $43 \%$ and $79 \%{ }^{9}$

\section{Cold Agglutinin Test}

This is a heterophile antibody test where IgM antibodies agglutinate various species of erythrocytes. The cold agglutinin test (CAT) is not a specific test for MP but is more easily available in India. Cold agglutinins are autoantibodies that agglutinate or clump human erythrocytes at $4^{\circ} \mathrm{C}$ but not at $37^{\circ} \mathrm{C} .^{12}$ Respiratory infections with MP evoke cold agglutinins (CAs), which can be an indirect evidence of MP infection. These are directed against the altered "I" antigens on the human erythrocyte surface in MP-infected patients. ${ }^{13}$ Cold agglutinins are the first antibodies to appear and also the first to disappear (6-8 weeks) in patients with MP infection. ${ }^{14} \mathrm{~A}$ titer of 1:32 is considered as positive.

\section{Advantage}

- Easily and widely available

\section{Disadvantages ${ }^{15}$}

- Cold agglutinins are seen in only $50-60 \%$ of MP infections and hence not reliable for diagnosis.

- False-positive CAT may be seen with other pathogens like $K$. pneumoniae, L. pneumophila, T. pallidum, influenza, adenovirus, Epstein-Barr virus, and Cytomegalovirus.

- False-positive CAT may also be seen in other diseases like hemolytic anemia, lymphoid cells malignancies, autoimmune diseases, and occasionally with antibiotic therapy.

\section{Other Nonspecific Tests}

Approximately one-third of patients may have leukocytosis with a raised erythrocyte sedimentation rate (ESR).$^{15}$ Lymphopenia has also been described in children with MP infection. ${ }^{16}$

\section{Conclusion}

M. pneumoniae is an important pathogen causing respiratory illness among children. Due to its nonspecific clinical features, it must be kept as a differential diagnosis to various other atypical bacteria and viruses known to cause respiratory disease. A high degree of clinical suspicion along with PCR-based testing in the 1st week helps early diagnosis. This should be supported by serology in the 2nd week of illness.

\section{References}

1. Atkinson TP, Balish MF, Waites KB. Epidemiology, clinical manifestations, pathogenesis and laboratory detection of Mycoplasma pneumoniae infections. FEMS Microbiol Rev 2008;32(6):956-973. DOI: 10.1111/j.1574-6976.2008.00129.x.

2. Waites KB, Xiao L, Paralanov V, et al. Molecular methods for the detection of Mycoplasma and Ureaplasma infections in humans: a paper from the 2011 William Beaumont hospital symposium on molecular pathology. J Molecul Diagnost 2012;14(5):437-450. DOI: 10.1016/j.jmoldx.2012.06.001.

3. Waites KB, Talkington DF. Mycoplasma pneumoniae and its role as a human pathogen. Clin Microbiol Rev 2004;17(4):697-728. DOI: 10.1128/CMR.17.4.697-728.2004.

4. Søndergaard MJ, Friis MB, Hansen DS, et al. Clinical manifestations in infants and children with Mycoplasma pneumoniae infection. PLoS ONE 2018;13(4):e0195288. DOI: 10.1371/journal.pone. 0195288.

5. Waites KB, Xiao L, Liu Y, et al. Mycoplasma pneumoniae from the respiratory tract and beyond. Clin Microbiol Rev 2017;30(3):747-809. DOI: 10.1128/CMR.00114-16.

6. Narita M. Pathogenesis of extrapulmonary manifestations of Mycoplasma pneumoniae infection with special reference to pneumonia. J Infect Chemother 2010;16(3):162-169. DOI: 10.1007/ s10156-010-0044-x.

7. Daxboeck F, Krause R, Wenisch C. Laboratory diagnosis of Mycoplasma pneumoniae infection. Clin Microbiol Infect 2003;9(4):263-273. DOI: 10.1046/j.1469-0691.2003.00590.x.

8. Loens K, Goossens H, leven M. Acute respiratory infection due to Mycoplasma pneumoniae: current status of diagnostic methods. Eur J Clin Microbiol Infect Dis 2010;29:1055-1069. DOI: 10.1007/s10096010-0975-2.

9. Thurman KA, Walter ND, Schwartz SB, et al. Comparison of laboratory diagnostic procedures for detection of Mycoplasma pneumoniae in community outbreaks. Clin Infect Dis 2009;48(9):1244-1249. DOI: $10.1086 / 597775$.

10. Miyashita N, Kawai $Y$, Tanaka T, et al. Diagnostic sensitivity of a rapid antigen test for the detection of Mycoplasma pneumoniae: comparison with real-time PCR. J Infect Chemother 2015;21(6):473475. DOI: 10.1016/j.jiac.2015.02.007.

11. Kumar S. Mycoplasma pneumoniae: a significant but underrated pathogen in paediatric community-acquired lower respiratory tract infections. Indian J Med Res 2018;147(1):23-31. DOI: 10.4103/ijmr. IJMR_1582_16.

12. Wijesooriya W, Sunil-Chandra N, Perera J. Reliability of cold agglutinin test (CAT) for the detection of patients with Mycoplasma pneumoniae pneumonia. Sri Lankan J Infect Dis 2016;6:25. DOI: 10.4038/sljid. v6i1.8099.

13. Swiecicki PL, Hegerova LT, Gertz MA. Cold agglutinin disease. Blood 2013;122(7):1114-1121. DOI: 10.1182/blood-2013-02-474437.

14. Khan FY, Yassin. MA. Mycoplasma pneumoniae associated with severe autoimmune hemolytic anemia: case report and literature review. Braz J Infect Dis 2009;13(1):77-79. DOI: 10.1590/S141386702009000100018.

15. Alves Vervloet L, Marguet C, Augusto P, et al. Infection by Mycoplasma pneumoniae and its importance as an etiological agent in childhood community-acquired pneumonias. Braz J Infect Dis 2007;11(5):507514. DOI: 10.1590/S1413-86702007000500012.

16. Youn YS, Lee KY, Hwang JY, et al. Difference of clinical features in childhood Mycoplasma pneumoniae pneumonia. BMC Pediatr 2010;10(1):48. DOI: 10.1186/1471-2431-10-48. 